\title{
Predicting Working Memory Training Benefits From Transcranial Direct Current Stimulation Using Resting-State fMRI
}

\author{
Adelle G. B. Cerreta ${ }^{1}$, Ryan E. B. Mruczek ${ }^{2}$ and Marian E. Berryhill ${ }^{1 *}$ \\ ${ }^{1}$ Program in Cognitive and Brain Sciences, Program in Integrative Neuroscience, Department of Psychology, University of \\ Nevada, Reno, NV, United States, ${ }^{2}$ Department of Psychology, College of the Holy Cross, Worcester, MA, United States
}

The effects of transcranial direct current stimulation (tDCS) on working memory (WM) performance are promising but variable and contested. In particular, designs involving one session of tDCS are prone to variable outcomes with notable effects of individual differences. Some participants benefit, whereas others are impaired by the same tDCS protocol. In contrast, protocols including multiple sessions of tDCS more consistently report WM improvement across participants. The objective of the current project was to

OPEN ACCESS

Edited by:

Francesca Marina Bosco,

University of Turin, Italy

Reviewed by:

Stevan Nikolin,

Black Dog Institute, Australia

Lysianne Beynel,

Duke University, United States

*Correspondence:

Marian E. Berryhill

mberryhill@unr.edu

Specialty section:

This article was submitted to

Cognitive Science,

a section of the journal

Frontiers in Psychology

Received: 05 June 2020 Accepted: 09 September 2020

Published: 14 October 2020

Citation:

Cerreta AGB, Mruczek REB and

Berryhill ME (2020) Predicting

Working Memory Training Benefits

From Transcranial Direct Current Stimulation Using Resting-State FMRI.

Front. Psychol. 11:570030.

doi: 10.3389/fpsyg.2020.570030 test whether differences in resting-state connectivity between stimulation site and two WM-relevant networks [default mode network (DMN) and central executive network (CEN)] could account for initial and longitudinal responses to tDCS. Healthy young adults completed 5 days of visual WM training during sham or anodal right frontal tDCS. The behavioral data showed that only the active tDCS group significantly improved over the visual WM training period. There were no significant correlations between initial response to tDCS and resting-state activity. DMN activity in the anterior cingulate cortex significantly correlated with WM training slope. These data underscore the importance of sampling in studies applying tDCS; homogeneity (e.g., of gender, special population, and WM capacity) may produce more consistent data in a single experiment with limited power, whereas heterogeneity is important in determining the mechanism(s) and potential for tDCS-linked protocols. This issue is a limitation in tDCS findings that continues to hamper its optimization and translational value.

Keywords: working memory, transcranial direct current stimulation, resting-state, fMRI, cognitive training

\section{INTRODUCTION}

Neurostimulation offers hope as a way to improve capacity-limited working memory (WM; Cowan, 2001) and its age-related decline (Reuter-Lorenz and Park, 2010). To date, cognitive training regimens produce modest results with little evidence of transfer to untrained tasks (for reviews see: Morrison and Chein, 2011; Shipstead et al., 2012; Melby-Lervåg et al., 2016; Simons et al., 2016; Katz et al., 2018; Redick, 2019; Sala and Gobet, 2019; Teixeira-Santos et al., 2019; but see: Au et al., 2016a). 
When WM benefits are found, the underlying mechanism appears to be enhanced frontoparietal connectivity (McNab and Klingberg, 2008; Constantinidis and Klingberg, 2016; Chen et al., 2019). These networks can be targeted using noninvasive transcranial direct current stimulation (tDCS). Some evidence shows that one session of $\mathrm{tDCS}$ can improve WM performance in healthy (Sandrini et al., 2012; Brunoni and Vanderhasselt, 2014; Trumbo et al., 2016; Brunye et al., 2017), and patient populations (Boggio et al., 2006; Jo et al., 2009; Saunders et al., 2015; Wu et al., 2016), reviewed in Berryhill and Martin (2018). In contrast to the variability in single-session protocols, tDCS-WM training protocols show more consistent WM benefits (Martin et al., 2013; Richmond et al., 2014; Jones et al., 2015b, 2017; Au et al., 2016b; Stephens and Berryhill, 2016; Katz et al., 2017; Ke et al., 2019; but see: Möller et al., 2017). However, the jury remains split as several reviews, and metaanalyses provide differing conclusions, suggesting that there are few cognitive benefits associated with tDCS (Horvath et al., 2015), that meta-analyses minimizing tDCS-effects are flawed (Antal et al., 2015), or that there are benefits under certain conditions, such as longitudinal designs (Brunoni and Vanderhasselt, 2014; Elmasry et al., 2015; Hill et al., 2016; Jantz et al., 2016; Mancuso et al., 2016).

We reiterate our stance that one underlying challenge is that individual differences play an important, but poorly understood, role in determining how a participant responds to tDCS. Individual participants show different behavioral effects in response to the same tDCS protocol (Benwell et al., 2015; Brunye et al., 2015; London and Slagter, 2015; Filmer et al., 2019). We previously reported that tDCS effects interacted with individual differences based on participants' WM capacity (Jones and Berryhill, 2012; Gözenman and Berryhill, 2016; Arciniega et al., 2018), level of educational attainment (Berryhill and Jones, 2012), and even level of motivation (Jones et al., 2015a). Others find that the temporal spacing of sessions ( $\mathrm{Au}$ et al., 2016b; Katz et al., 2017), brain morphology (Kim et al., 2014), and even genetics (Wiegand et al., 2016) modulate tDCS effects. A few studies examining individual differences in longitudinal designs find evidence of different responses even after multiple sessions (Stephens et al., 2017; Talsma et al., 2017; Ke et al., 2019). Individual differences appear to be more consequential in single-session compared to multiple-session tDCS studies. One persistent difficulty is to sufficiently understand the underlying mechanism of tDCS-linked WM benefits to leverage these differences into tailored, efficacious protocols.

Here, we test the hypothesis that initial resting-state connectivity will predict the initial response as well as the longitudinal response to tDCS in a visual WM task. This hypothesis builds on prior findings showing that tDCS alters synaptic strength in task-relevant networks (Filmer et al., 2014) for a period of time after stimulation (Callan et al., 2016; Jones et al., 2017; Möller et al., 2017; Antonenko et al., 2018; Fiori et al., 2018; Nissim et al., 2019). Furthermore, tDCS targeting the right prefrontal cortex (PFC) revealed strengthened functional connectivity in frontoparietal networks (Peña-Gómez et al., 2012) within the central executive network (CEN; Collette and Van der Linden, 2002; Owen et al., 2005; Seeley et al., 2007;
Bressler and Menon, 2010; Peña-Gómez et al., 2012) and other cortical-subcortical networks (Polania et al., 2011). The CEN is a task-positive network with stronger connectivity between dorsolateral PFC (DLPFC) and posterior parietal cortex associated with superior WM performance (Duncan and Owen, 2000; Curtis and D'Esposito, 2003; Bunge and Wright, 2007; Palva et al., 2010). In addition, connectivity in the task-negative default mode network (DMN) is important for WM performance (Hampson et al., 2010; Sambataro et al., 2010; Vatansever et al., 2017) and can predict individual differences in WM performance (Sala-Llonch et al., 2012). tDCS can also modulate DMN connectivity (Keeser et al., 2011; Abellaneda-Perez et al., 2019; Chase et al., 2019; Donaldson et al., 2019; Mezger et al., 2019). Finally, to complete the circle on these two networks, the relationship between the CEN and DMN is related to WM performance (Hampson et al., 2010).

Collectively, these findings raise the possibility that an individual's pattern of connectivity may predict their response to tDCS. We collected baseline resting-state fMRI (rsfMRI) and conducted a tDCS-WM training study in the same individuals. We predicted that we would replicate the observation that stronger resting-state connectivity in the DMN or CEN would predict higher WM capacity before tDCS. The next prediction related to initial response to tDCS. Our logic was that the subtle effect of one tDCS session would be enough to modulate well-functioning networks in those with higher WM capacity and stronger connectivity. Whereas in low WM capacity participants, we anticipated insufficient connectivity that would require multiple tDCS sessions to improve WM performance. The prediction associated with the more consistent response associated with longitudinal response was that there might be distinct pattern of connectivity that collectively predicted performance gains over multiple tDCS sessions.

\section{MATERIALS AND METHODS}

\section{Participants}

Forty-six University of Nevada, Reno students participated in exchange for their choice of $\$ 15 / \mathrm{h}$ or course credit (active tDCS: $N=28$, ages 18-36, $M=22.7,12$ females; sham: $N=18$ ages $18-32, M=21.4$, eight females). Participants had no history of neurological conditions and were not taking any sedative-hypnotic medications that might alter neural excitability. All protocols were approved by the Internal Review Board of the University of Nevada, and informed consent was collected prior to participation. The data from three participants in the active tDCS group were excluded: one participant due to below chance $(<50 \%)$ performance across all sessions and two others for failing to complete all five sessions.

\section{Resting-State MRI Acquisition and Preprocessing}

First, participants completed one rsfMRI session prior to WM testing or any tDCS. Participants kept eyes closed during 2-3 rsfMRI runs ( $\sim 5.3 \mathrm{~min}$ each); 14 active group members completed two runs, and the remaining participants completed three runs. 
Images were acquired on a 3 T Philips (Andover, MA, United States) MRI scanner with an eight-channel SENSE parallel head coil. A set of $155 \mathrm{~T} 2{ }^{*}$-weighted volumes were obtained [repetition time $(T R)=2,000 \mathrm{~ms}$, echo time $(T E)=30 \mathrm{~ms}, 32$ slices per volumes, slice thickness $=3 \mathrm{~mm}$, field of view $(\mathrm{FOV})=240 \mathrm{~mm}^{2}$, matrix size $128 \times 128$, in-plane resolution $=1.875 \mathrm{~mm}]$. Functional data were aligned to a high-resolution $3 \mathrm{D}$ structural dataset using an echo-planar 3D T1-weighted image.

Preprocessing was completed in analysis of functional neuroimages (AFNI; Cox, 1996; http://afni.nimh.nih.gov/afni/), SUMA (Saad et al., 2004; http://afni.nimh.nih.gov/afni/suma/), and FreeSurfer (Dale et al., 1999; Fischl et al., 1999; http://surfer. nmr.mgh.harvard.edu/) using the standardized script afni_proc. $p y .{ }^{1}$ The first two TRs were removed, then the data were despiked, and slice-time and motion were corrected, as well as spatially normalized to an Montreal Neurological Institute (MNI) template. Bandpass filtering was employed to temporally filter and retain frequencies between 0.01 and $0.1 \mathrm{~Hz}$. Censoring was based on motion parameters and signal outliers within the BOLD data (Power et al., 2014, 2015). Six motion parameter estimates, ventricular and white matter signals, and baseline, linear, quadratic, and cubic trends were removed by linear regression (Fox et al., 2009).

\section{Regions of Interest and Producing Seeds}

Seeds were restricted to right hemispheric areas, as we used spatial WM tasks. Seed regions of interest (ROIs) were manually generated in AFNI. The seeds were $12 \mathrm{~mm}$ spheres to allow for broader connectivity maps and to be consistent with existing guidelines (Fox et al., 2005). The following seed locations were selected: (1) right DLPFC, a node in the CEN for correlations related to executive functioning during resting-state (MNI coordinates: 44, 36, and 20; Seeley et al., 2007); and (2) right posterior cingulate cortex (PCC) within the DMN to acquire canonical resting-state activity maps (MNI coordinates: 5, -49, and 40; Fox et al., 2005).

\section{Resting-State Analysis}

To determine connectivity strength between these seed regions and the rest of the brain, AFNI's 3dUndump created the ROI by generating a $3 \mathrm{D}$ dataset from the specified coordinates. $3 d$ maskave was used to generate the time course of activity in the given seed region. To determine correlation values, $3 \mathrm{dfim}+$ was used to correlate time course correlations within either the DMN seed (right PCC) or CEN seed (right DLPFC) and behavioral performance. $3 d f i m+$ was also used to correlate the DMN and CEN time courses with time courses throughout the whole brain, generating resting-state connectivity maps of Pearson's $r$ values. Pearson's $r$ values were converted to $z$-scores using Fisher's $r$-to- $z$ transformation and the expression “ $\log [(1+a) /(1-a)] / 2$." In SPSS (version 24, IBM, Armonk, NY, United States), $z$-scores, and performance scores for each individual were compared across all participants, as well as by high and low WM capacity groups (see below), to identify significant differences in hemodynamic activity and performance.

${ }^{1}$ http://afni.nimh.nih.gov/pub/dist/doc/program_help/afni_proc.py.html

\section{Transcranial Direct Current Stimulation}

Anodal tDCS stimulation was applied via two saline-dampened scalp electrodes $\left(5 \times 7 \mathrm{~cm}^{2}\right)$ and delivered at $2 \mathrm{~mA}$ for $20 \mathrm{~min}$ by a continuous current stimulator (neuroConn DC Stimulator, $\mathrm{GmbH}$, Germany). The anode was centered over the right (F4) DLPFC. The cathode was placed on the contralateral cheek (Berryhill et al., 2010; Jones et al., 2015b). Sham stimulation also involved placement of electrodes in these areas, but unbeknownst to the participant, only brief (20 s ramp up/down) stimulation was applied. Current modeling was also investigated using the Realistic vOlumetric Approach to Simulate Transcranial Electric Stimulation (ROAST, Huang et al., 2019; see Figure 1).

\section{Behavioral Paradigms}

On Day 1 of the five-session WM training period, participants completed the Automated Operation Span (OSPAN) to provide an independent baseline measure of WM performance (Unsworth et al., 2005), prior to any tDCS. Participants remembered the order of a string of sequentially presented letters interleaved with arithmetic problems that also required WM.

Participants received tDCS during WM training tasks performance. Participants completed three tasks: a change detection task (set sizes 6 and 8) and a two-back visuospatial task (described below). In the change detection tasks, participants viewed sixor eight-colored squares $\left(250 \mathrm{~ms}, 3 \times 3^{\circ}\right.$, white, black, red, pink, orange, yellow, green, teal, blue, aqua, and purple; Jones and Berryhill, 2012). After a delay (1,000 ms) a probe image $(2,200 \mathrm{~ms})$ appeared that was unchanged from encoding $(50 \%)$ or included one color change (50\%). Participants responded via key press ("o" match and " $n$ " mismatch). There were 100 trials per set size, and the task lasted $\sim 10 \mathrm{~min}$. The change detection task was used because although attention is needed for the task, WM is crucial for accessing the representation stored during encoding for comparison. In the spatial $n$-back task participants

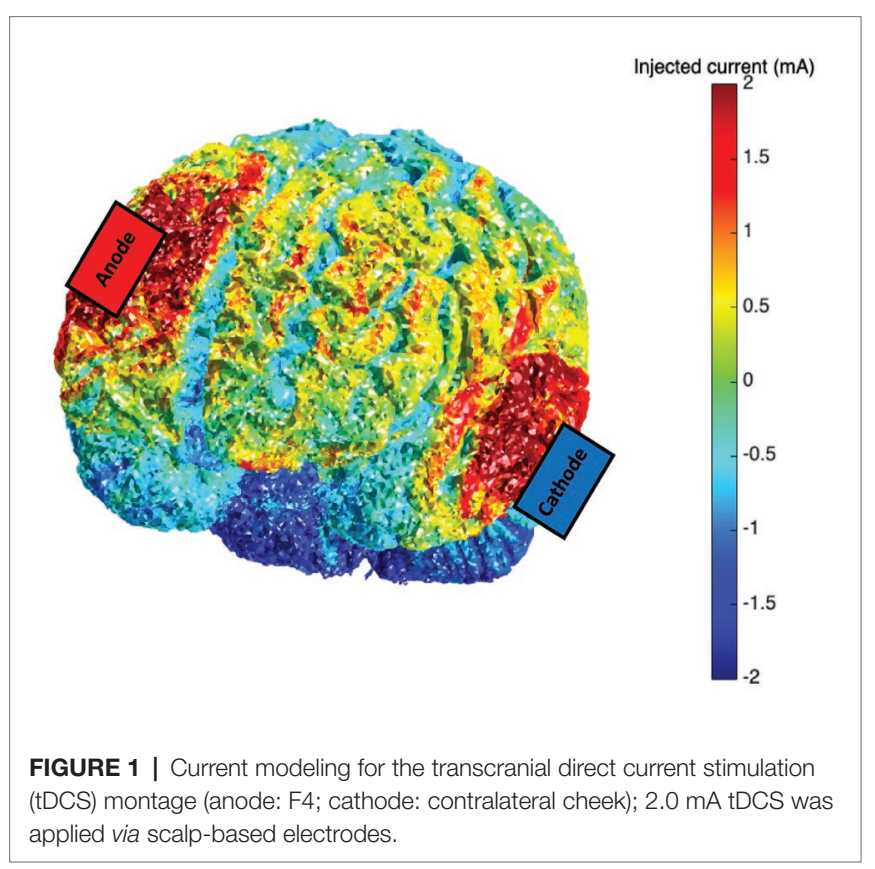


monitored the location of a green circle (500 ms) across nine possible locations (Stephens and Berryhill, 2016). During each presentation, participants reported via key press (“j” match and "f" mismatch) whether the current location matched the location occupied two presentations previously. Participants completed 450 trials (150 match and 300 no match) in $\sim 10 \mathrm{~min}$.

The primary measure for each task was percent accuracy. Percent accuracy on the two-back task was calculated for each day individually, with analyses geared toward revealing any significant differences in percent accuracy dependent on session number. The same analyses were completed for the change detection task with set sizes 6 and 8, individually. Subsequently, performance slope over the five sessions was also considered to further investigate improvement.

\section{RESULTS}

\section{The Active-tDCS Group Improved on the Two-Back Task With Training}

The first question to address was to determine whether active tDCS significantly changed WM performance compared to sham stimulation. We first conducted separate repeated measures ANOVAs with the factors of group (active tDCS and sham) and session (Days 1-5) for each WM training task. This revealed that neither group significantly improved on either of the change detection tasks [set sizes 6 and 8; set size 6: $F(4,168)=2.04$, $p=0.09, \eta_{\mathrm{p}}{ }^{2}=0.05$; set size 8: $F(4,164)=0.96, p=0.43$, $\left.\eta_{\mathrm{p}}{ }^{2}=0.02\right]$. Performance on the change detection task was poor and varied little across sessions (set size 6: active: Day 1: mean, SD: 65.68, 7.95\%; Day 5: 68.20, 7.37\%; sham: 66.00, 7.39\%, Day 5: 69.18, 7.04\%; set size 8: active: Day 1: 62.16, 6.44\%; Day 5: 62.84, 7.15\%; sham: Day 1: 61.13, 7.04\%; Day 5: $63.06,4.52 \%)$. However, performance on the two-back task revealed a significant group $\times$ session interaction indicating that only the active group significantly improved across sessions $\left[F(4.88,75.66)=7.15, p<0.001, \eta_{p}{ }^{2}=0.16\right.$; Greenhouse-Geisser correction applied for violation of sphericity; see Figure 2, but this effect did not survive when performance on Day 1 was entered as a covariate $F(1,40)=0.54, p=0.47]$. A complementary analysis of slope across training sessions provided

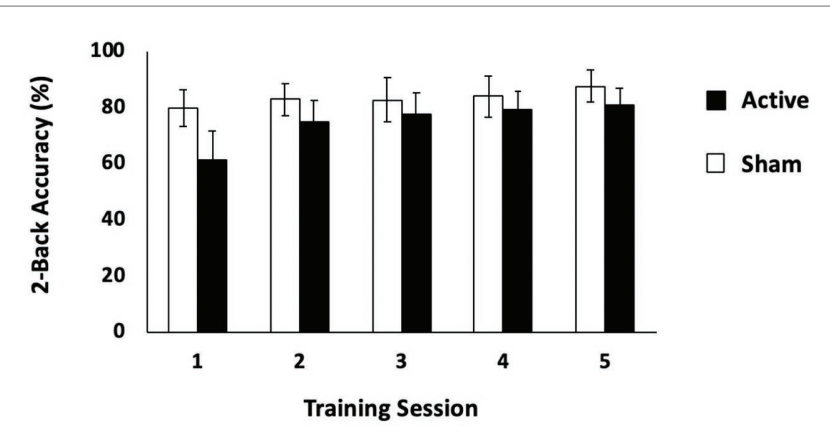

FIGURE 2 | Behavioral results: two-back task. Performance (\% correct) on the two-back task for the active and sham tDCS stimulation conditions. Error bars represent $95 \%$ Cls. confirmation that the active tDCS group improved more steeply than did the sham group [sham: mean slope $(M)=0.06$, $95 \% C I=(-0.47,0.60)$; active: $M=3.36, C I=(1.75,4.97)$; independent-samples $t$-test, $t(31.70)=2.96, p=0.006, \eta^{2}=0.23$; Levene's test: $p=0.004]$. This effect did survive an ANCOVA with Day 1 performance as a covariate $[F(1,40)=7.00, p=0.012]$. In sum, only the active tDCS group showed significant improvement in WM performance over the 5 days of training.

Reaction times on the two-back task were investigated, but no significant difference in reaction time performance was found between active and sham groups [Greenhouse-Geisser corrected, $F(2.146,87.968)=0.10, p=0.92]$.

\section{Active tDCS Group: Predicting Training Response to tDCS Resting-State fMRI Does Not Predict Initial Response to tDCS (Day 1 Response)}

With regard to the rsfMRI data, we were interested in the relationship between pre-existing rsfMRI and an individual's WM response to initial and longitudinal tDCS. We planned on probing rsfMRI within the DMN and CEN to test whether connectivity in these networks predicted the initial and/or later responsivity to tDCS. The logic was that this might be useful in predicting tDCS-linked WM performance. There were no significant correlations reflecting initial response to tDCS on Day 1 of WM training (two-back task) and either rsfMRI seed. In other words, we found no support for our hypothesis that resting-state differences in connectivity would predict initial response to $\mathrm{tDCS}$.

\section{Resting-State fMRI Does Predict Longitudinal tDCS Response (Training Slope)}

In the active tDCS group, we investigated whether rsfMRI predicted WM performance across tDCS-linked WM training. To address this question, we sought correlations between training slope on the two-back task and rsfMRI correlations with two networks, the DMN, and CEN. For the CEN seed, there was no significant correlation that predicted WM performance slope. However, the connectivity between the DMN seed (PCC) and the anterior cingulate cortex positively correlated with WM performance slope for the active group [Pearson's $r(25)=0.39$, $p=0.05$, MNI coordinates: $(4,12$, and 36); see Figure 3], but not for the sham group $[r(18)=0.25, p=0.31]$. DMN connectivity before training predicted active group gains in the 2-back task.

\section{DISCUSSION}

In this experiment, WM training, tDCS, and rsfMRI measures were combined to clarify the basis of individual differences in tDCS-linked WM response. We tested whether pre-existing connectivity predicted initial or longitudinal responses to tDCSlinked cognitive performance. Participants completed change detection and $n$-back WM tasks across five training sessions with or without active right frontal tDCS. The data showed that 

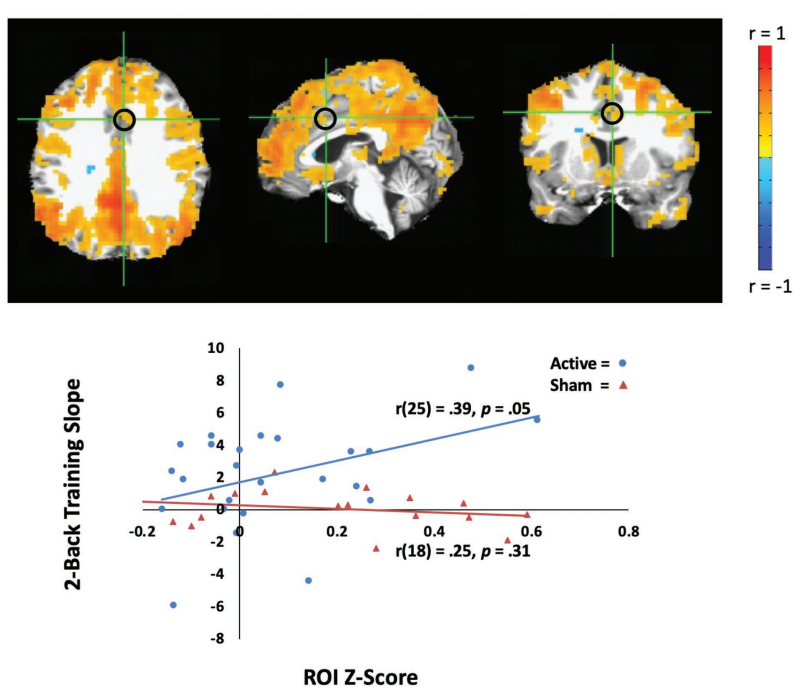

FIGURE 3 | Resting-state fMRI (rsfMRI) activity correlates with behavior learning across the training in the active tDCS group. Top: The average of default mode network (DMN) activity (whole brain connectivity generated from the PCC seed and circled in black) in the active tDCS group participants. The left hemisphere is shown on the left. Bottom: Training slope on the two-back task significantly correlated with DMN activity in the anterior cingulate cortex [Montreal Neurological Institute (MNI) seed: (4, 12, and 36), black circle]. The sham group participants are shown as orange triangles, and the active group participants are shown as blue circles.

only the active tDCS group significantly improved. Improvement was isolated to performance on the two-back task as performance on the change detection task was consistent across sessions.

Notably, there were no patterns of resting-state connectivity that corresponded with Day 1 responses to tDCS. This held across both seed locations, one in the DMN and one in the CEN. These data provide no support for our intuition that connectivity could characterize the effects of individual differences after a single session of tDCS. As noted above, we have previously seen data that conform to a phenomenon of the "rich get richer" such that performance improvements occur in the subset of participants with high WM capacity (Jones and Berryhill, 2012) or high education level (Berryhill and Jones, 2012). If existing connectivity patterns do not explain initial responses to tDCS, we speculate that neural excitability during tDCS explains immediate effects. In short, it has been found that those who show greater increase in BOLD during anodal parietal tDCS demonstrate superior performance in a visual navigation task (Falcone et al., 2018). Alternatively, the effect of connectivity may be quite small, and only detected with more participants, or higher resolution scanning.

In contrast, two-back WM training slope was predicted by the coupling strength between the DMN seed and the anterior cingulate cortex. After WM training, resting-state scans have shown that the anterior cingulate cortex exhibits increased functional connectivity with frontoparietal networks, with personality indices correlating with changes in anterior cingulate cortex activity (Gray and Braver, 2002; Jolles et al., 2013). Lesions to this area impair WM ability in identifying errors and executing a corrective action (Rushworth et al., 2003). Our interpretation is that the tDCS builds on existing connectivity to further improve WM performance. It predicts that individuals with well-developed connectivity between these areas may benefit more from longitudinal tDCS than those with less. In other words, the current analyses may have identified the tips of the icebergs, and further work is needed to clarify those who will benefit from tDCS.

Assuredly, with more rsfMRI scans and more participants, we may be better able to identify more subtle relationships. It is now quite clear that tDCS provides a modest neuromodulatory effect that serves as a tipping factor to alter neural firing. A major challenge in going forward in developing effective tDCS protocols for cognition or clinical populations is that in addition to individual differences such as connectivity, there are many factors and stimulation parameters that predict an individual's response to tDCS. These variables include neural excitability, amount of sleep, engagement with the task, genotype (see Katz et al., 2017 for review).

\section{LIMITATIONS}

A major limitation is that our participants exhibited low heterogeneity in their WM performance than in our participant samples in previous studies. Because we were conducting resting-state scans paired with a longitudinal tDCS design, we worried about attrition, and consequently, we recruited from department affiliates (e.g., graduate students and research assistants). This meant that we were drawing from a homogeneous pool and a group that was strongly motivated to provide quality data. Thus, solving one problem raised a second, unanticipated challenge: less range in our participants' performance, especially in the sham group. Additional differences in connectivity might be more nuanced or clearer in a more representative population. In addition, connectivity patterns in older adults change over time (Chan et al., 2014), and what may be useful in predicting tDCS responsiveness in young adults may differ in the elderly. These issues served to weaken our statistics and render null the difference between the active and sham group correlations.

A second limitation that reduced generalizability was that the change detection tasks showed no improvement across sessions, possibly due to a lack of engagement with the task and leading us to eliminate these data from further analyses. We implemented multiple tasks to address generality of any observed effects, and we did not want our participants to be bored with many trials of the same task over multiple days. Finally, we would like to have been able to collect more resting-state scans per person to have cleaner, more powerful data. A major advantage to using rsfMRI over task-related fMRI is the reduced time and number of scans necessary compared to when typically using a task. More subjects can be used, and sensitive subjects do not have to be in the scanner for as long. An additional possibility is that despite existing findings associating rsfMRI with task performance, it may not be as sensitive to subtle changes elicited by tDCS. In other words, task-related fMRI may be superior in these situations. Future work with more powerful rsfMRI 
protocols is essential in building the optimized, tailored tDCS protocol to maximize performance in an efficient manner.

\section{OPEN QUESTIONS}

TDCS may yet prove to be a generally useful translational approach. Converging evidence supports the importance of neural synchronization to change neural networks during WM task performance. A major challenge is to determine a priori who respond to stimulation. Individual differences are an acknowledged aspect of the noninvasive stimulation literature and help to explain inconsistent results. Converging evidence from various techniques, including further testing of tDCS parameters, morphological and functional differences in the brain, and genetic differences are needed.

\section{DATA AVAILABILITY STATEMENT}

The raw data supporting the conclusions of this article will be made available by the authors, without undue reservation.

\section{REFERENCES}

Abellaneda-Perez, K., Vaque-Alcazar, L., Perellon-Alfonso, R., Bargallo, N., Kuo, M. F., Pascual-Leone, A., et al. (2019). Differential tDCS and tACS effects on working memory-related neural activity and resting-state connectivity. Front. Neurosci. 13:1440. doi: 10.3389/fnins.2019.01440

Antal, A., Keeser, D., Priori, A., Padberg, F., and Nitsche, M. A. (2015). Conceptual and procedural shortcomings of the systematic review "evidence that transcranial direct current stimulation (tDCS) generates little-to-no reliable neurophysiologic effect beyond mep amplitude modulation in healthy human subjects: a systematic review" by Horvath and co-workers. Brain Stimul. 8, 846-849. doi: 10.1016/j.brs.2015.05.010

Antonenko, D., Nierhaus, T., Meinzer, M., Prehn, K., Thielscher, A., Ittermann, B., et al. (2018). Age-dependent effects of brain stimulation on network centrality. NeuroImage 176, 71-82. doi: 10.1016/j.neuroimage.2018.04.038

Arciniega, H., Gozenman, F., Jones, K. T., Stephens, J. A., and Berryhill, M. E. (2018). Frontoparietal tDCS benefits visual working memory in older adults with low working memory capacity. Front. Aging Neurosci. 10:57. doi: 10.3389/ fnagi.2018.00057

Au, J., Buschkuehl, M., Duncan, G. J., and Jaeggi, S. M. (2016a). There is no convincing evidence that working memory training is NOT effective: a reply to Melby-Lervåg and Hulme (2015). Psychon. Bull. Rev. 23, 331-337. doi: 10.3758/s13423-015-0967-4

Au, J., Katz, B., Buschkuehl, M., Bunarjo, K., Senger, T., Zabel, C., et al. (2016b). Enhancing working memory training with transcranial direct current stimulation. J. Cogn. Neurosci. 28, 1419-1432. doi: 10.1162/jocn_a_00979

Benwell, C. S., Learmonth, G., Miniussi, C., Harvey, M., and Thut, G. (2015). Non-linear effects of transcranial direct current stimulation as a function of individual baseline performance: evidence from biparietal tDCS influence on lateralized attention bias. Cortex 69, 152-165. doi: 10.1016/j.cortex.2015.05.007

Berryhill, M. E., and Jones, K. T. (2012). tDCS selectively improves working memory in older adults with more education. Neurosci. Lett. 521, 148-151. doi: 10.1016/j.neulet.2012.05.074

Berryhill, M. E., and Martin, D. (2018). Cognitive effects of transcranial direct current stimulation in healthy and clinical populations: an overview. J. ECT 34, e25-e35. doi: 10.1097/YCT.0000000000000534

Berryhill, M. E., Wencil, E. B., Coslett, B. H., and Olson, I. R. (2010). A selective working memory impairment after transcranial direct current stimulation to the right parietal lobe. Neurosci. Lett. 479, 312-316. doi: 10.1016/j.neulet.2010.05.087

\section{ETHICS STATEMENT}

The studies involving human participants were reviewed and approved by University of Nevada, Reno IRB. The patients/ participants provided their written informed consent to participate in this study.

\section{AUTHOR CONTRIBUTIONS}

$\mathrm{AC}$ and $\mathrm{MB}$ designed the study. AC collected the data. AC and RM completed analyses. All authors wrote the manuscript. All authors contributed to the article and approved the submitted version.

\section{FUNDING}

This work was supported by generous funding from NIGMS 1P20GM103650 (Imaging CORE) and the National Science Foundation (NSF OIA 1632849 and NSF OIA 1632738) to MB.

Boggio, P. S., Ferrucci, R., Rigonatti, S. P., Covre, P., Nitsche, M., Pascual-Leone, A., et al. (2006). Effects of transcranial direct current stimulation on working memory in patients with Parkinson's disease. J. Neurol. Sci. 249, 31-38. doi: 10.1016/j.jns.2006.05.062

Bressler, S. L., and Menon, V. (2010). Large-scale brain networks in cognition: emerging methods and principles. Trends Cogn. Sci. 14, 277-290. doi: 10.1016/j.tics.2010.04.004

Brunoni, A. R., and Vanderhasselt, M. A. (2014). Working memory improvement with non-invasive brain stimulation of the dorsolateral prefrontal cortex: a systematic review and meta-analysis. Brain Cogn. 86, 1-9. doi: 10.1016/j. bandc.2014.01.008

Brunye, T. T., Moran, J. M., Cantelon, J., Holmes, A., Eddy, M. D., Mahoney, C. R., et al. (2015). Increasing breadth of semantic associations with left frontopolar direct current brain stimulation: a role for individual differences. Neuroreport 26, 296-301. doi: 10.1097/WNR.0000000000000348

Brunye, T. T., Moran, J. M., Holmes, A., Mahoney, C. R., and Taylor, H. A. (2017). Non-invasive brain stimulation targeting the right fusiform gyrus selectively increases working memory for faces. Brain Cogn. 113, 32-39. doi: 10.1016/j.bandc.2017.01.006

Bunge, S. A., and Wright, S. B. (2007). Neurodevelopmental changes in working memory and cognitive control. Curr. Opin. Neurobiol. 17, 243-250. doi: 10.1016/j.conb.2007.02.005

Callan, D. E., Falcone, B., Wada, A., and Parasuraman, R. (2016). Simultaneous tDCS-fMRI identifies resting state networks correlated with visual search enhancement. Front. Hum. Neurosci. 10:72. doi: 10.3389/fnhum.2016.00072

Chan, M. Y., Park, D. C., Savalia, N. K., Petersen, S. E., and Wig, G. S. (2014). Decreased segregation of brain systems across the healthy adult lifespan. Proc. Natl. Acad. Sci. U. S. A. 111, E4997-E5006. doi: 10.1073/ pnas.1415122111

Chase, H., Graur, S., Bertocci, M., and Phillips, M. (2019). Effect of cathodal transcranial direct current stimulation to the left ventrolateral prefrontal cortex on resting state default mode connectivity. Brain Stimul. 12, 472-473. doi: $10.1016 /$ j.brs.2018.12.540

Chen, C. C., Kuo, J. C., and Wang, W. J. (2019). Distinguishing the visual working memory training and practice effects by the effective connectivity during n-back tasks: a DCM of ERP study. Front. Behav. Neurosci. 13:84. doi: 10.3389/fnbeh.2019.00084

Collette, F., and Van der Linden, M. (2002). Brain imaging of the central executive component of working memory. Neurosci. Biobehav. Rev. 26, 105-125. doi: 10.1016/s0149-7634(01)00063-x 
Constantinidis, C., and Klingberg, T. (2016). The neuroscience of working memory capacity and training. Nat. Rev. Neurosci. 17, 438-449. doi: 10.1038/nrn.2016.43

Cowan, N. (2001). The magical number 4 in short-term memory: a reconsideration of mental storage capacity. Behav. Brain Sci. 24, 87-114. doi: 10.1017/ s0140525x01003922

Cox, R. W. (1996). AFNI: software for analysis and visualization of functional magnetic resonance neuroimages. Comput. Biomed. Res. 29, 162-173. doi: 10.1006/cbmr.1996.0014

Curtis, C. E., and D'Esposito, M. (2003). Persistent activity in the prefrontal cortex during working memory. Trends Cogn. Sci. 7, 415-423. doi: 10.1016/ s1364-6613(03)00197-9

Dale, A. M., Fischl, B., and Sereno, M. I. (1999). Cortical surface-based analysis. I. Segmentation and surface reconstruction. NeuroImage 9, 179-194. doi: 10.1006/nimg.1998.0395

Donaldson, P. H., Kirkovski, M., Yang, J. S., Bekkali, S., and Enticott, P. G. (2019). High-definition tDCS to the right temporoparietal junction modulates slow-wave resting state power and coherence in healthy adults. J. Neurophysiol. 122, 1735-1744. doi: 10.1152/jn.00338.2019

Duncan, J., and Owen, A. M. (2000). Common regions of the human frontal lobe recruited by diverse cognitive demands. Trends Neurosci. 23, 475-483. doi: 10.1016/s0166-2236(00)01633-7

Elmasry, J., Loo, C., and Martin, D. (2015). A systematic review of transcranial electrical stimulation combined with cognitive training. Restor. Neurol. Neurosci. 33, 263-278. doi: 10.3233/RNN-140473

Falcone, B., Wada, A., Parasuraman, R., and Callan, D. E. (2018). Individual differences in learning correlate with modulation of brain activity induced by transcranial direct current stimulation. PLoS One 13:e0197192. doi: 10.1371/journal.pone.0197192

Filmer, H. L., Dux, P. E., and Mattingley, J. B. (2014). Applications of transcranial direct current stimulation for understanding brain function. Trends Neurosci. 37, 742-753. doi: 10.1016/j.tins.2014.08.003

Filmer, H. L., Ehrhardt, S. E., Bollmann, S., Mattingley, J. B., and Dux, P. E. (2019). Accounting for individual differences in the response to tDCS with baseline levels of neurochemical excitability. Cortex 115, 324-334. doi: 10.1016/j.cortex.2019.02.012

Fiori, V., Kunz, L., Kuhnke, P., Marangolo, P., and Hartwigsen, G. (2018). Transcranial direct current stimulation (tDCS) facilitates verb learning by altering effective connectivity in the healthy brain. NeuroImage 181, 550-559. doi: 10.1016/j.neuroimage.2018.07.040

Fischl, B., Sereno, M. I., and Dale, A. M. (1999). Cortical surface-based analysis. II: inflation, flattening, and a surface-based coordinate system. NeuroImage 9, 195-207. doi: 10.1006/nimg.1998.0396

Fox, M. D., Snyder, A. Z., Vincent, J. L., Corbetta, M., Van Essen, D. C., and Raichle, M. E. (2005). The human brain is intrinsically organized into dynamic, anticorrelated functional networks. Proc. Natl. Acad. Sci. U. S. A. 102, 9673-9678. doi: 10.1073/pnas.0504136102

Fox, M. D., Zhang, D., Snyder, A. Z., and Raichle, M. E. (2009). The global signal and observed anticorrelated resting state brain networks. J. Neurophysiol. 101, 3270-3283. doi: 10.1152/jn.90777.2008

Gözenman, F., and Berryhill, M. E. (2016). Working memory capacity differentially influences responses to tDCS and HD-tDCS in a retro-cue task. Neurosci. Lett. 629, 105-109. doi: 10.1016/j.neulet.2016.06.056

Gray, J. R., and Braver, T. S. (2002). Personality predicts working-memoryrelated activation in the caudal anterior cingulate cortex. Cogn. Affect. Behav. Neurosci. 2, 64-75. doi: 10.3758/cabn.2.1.64

Hampson, M., Driesen, N., Roth, J. K., Gore, J. C., and Constable, R. T. (2010). Functional connectivity between task-positive and task-negative brain areas and its relation to working memory performance. Magn. Reson. Imaging 28, 1051-1057. doi: 10.1016/j.mri.2010.03.021

Hill, A. T., Fitzgerald, P. B., and Hoy, K. E. (2016). Effects of anodal transcranial direct current stimulation on working memory: a systematic review and meta-analysis of findings from healthy and neuropsychiatric populations. Brain Stimul. 9, 197-208. doi: 10.1016/j.brs.2015.10.006

Horvath, J. C., Forte, J. D., and Carter, O. (2015). Quantitative review finds no evidence of cognitive effects in healthy populations from single-session transcranial direct current stimulation (tDCS). Brain Stimul. 8, 535-550. doi: 10.1016/j.brs.2015.01.400

Huang, Y., Datta, A., Bikson, M., and Parra, L. C. (2019). Realistic volumetricapproach to simulate transcranial electric stimulation-ROAST-a fully automated open-source pipeline. J. Neural Eng. 16:056006. doi: 10.1088/1741-2552/ab208d
Jantz, T. K., Katz, B., and Reuter-Lorenz, P. A. (2016). Uncertainty and promise: the effects of transcranial direct current stimulation on working memory. Curr. Behav. Neurosci. Rep. 3, 109-121. doi: 10.1007/s40473-016-0071-8

Jo, J. M., Kim, Y. H., Ko, M. H., Ohn, S. H., Joen, B., and Lee, K. H. (2009). Enhancing the working memory of stroke patients using tDCS. Am. J. Phys. Med. Rehabil. 88, 404-409. doi: 10.1097/PHM.0b013e3181a0e4cb

Jolles, D. D., van Buchem, M. A., Crone, E. A., and Rombouts, S. A. (2013). Functional brain connectivity at rest changes after working memory training. Hum. Brain Mapp. 34, 396-406. doi: 10.1002/hbm.21444

Jones, K. T., and Berryhill, M. E. (2012). Parietal contributions to visual working memory depend on task difficulty. Front. Psychiatry 3:81. doi: 10.3389/ fpsyt.2012.00081

Jones, K. T., Gözenman, F., and Berryhill, M. E. (2015a). The strategy and motivational influences on the beneficial effect of neurostimulation: a tDCS and fNIRS study. NeuroImage 105, 238-247. doi: 10.1016/j.neuroimage.2014.11.012

Jones, K. T., Peterson, D. J., Blacker, K. J., and Berryhill, M. E. (2017). Frontoparietal neurostimulation modulates working memory training benefits and oscillatory synchronization. Brain Res. 1667, 28-40. doi: 10.1016/j. brainres.2017.05.005

Jones, K. T., Stephens, J. A., Alam, M., Bikson, M., and Berryhill, M. E. (2015b). Longitudinal neurostimulation in older adults improves working memory. PLoS One 10:e121904. doi: 10.1371/journal.pone.0121904

Katz, B., Au, J., Buschkuehl, M., Abagis, T., Zabel, C., Jaeggi, S. M., et al. (2017). Individual differences and long-term consequences of tDCS-augmented cognitive training. J. Cogn. Neurosci. 29, 1498-1508. doi: 10.1162/jocn_a_01115

Katz, B., Shah, P., and Meyer, D. E. (2018). How to play 20 questions with nature and lose: reflections on 100 years of brain-training research. Proc. Natl. Acad. Sci. U. S. A. 115, 9897-9904. doi: 10.1073/pnas.1617102114

Ke, Y., Wang, N., Du, J., Kong, L., Liu, S., Xu, M., et al. (2019). The effects of transcranial direct current stimulation (tDCS) on working memory training in healthy young adults. Front. Hum. Neurosci. 13:19. doi: 10.3389/ fnhum.2019.00019

Keeser, D., Meindl, T., Bor, J., Palm, U., Pogarell, O., Mulert, C., et al. (2011). Prefrontal transcranial direct current stimulation changes connectivity of resting-state networks during fMRI. J. Neurosci. 31, 15284-15293. doi: 10.1523/ JNEUROSCI.0542-11.2011

Kim, J. H., Kim, D. W., Chang, W. H., Kim, Y. H., Kim, K., and Im, C. H. (2014). Inconsistent outcomes of transcranial direct current stimulation may originate from anatomical differences among individuals: electric field simulation using individual MRI data. Neurosci. Lett. 564, 6-10. doi: 10.1016/j. neulet.2014.01.054

London, R. E., and Slagter, H. A. (2015). Effects of transcranial direct current stimulation over left dorsolateral $\mathrm{pFC}$ on the attentional blink depend on individual baseline performance. J. Cogn. Neurosci. 27, 2382-2393. doi: 10.1162/jocn_a_00867

Mancuso, L. E., Ilieva, I. P., Hamilton, R. H., and Farah, M. J. (2016). Does transcranial direct current stimulation improve healthy working memory?: a meta-analytic review. J. Cogn. Neurosci. 28, 1063-1089. doi: 10.1162/jocn_a_00956

Martin, D. M., Liu, R., Alonzo, A., Green, M., Player, M. J., Sachdev, P., et al. (2013). Can transcranial direct current stimulation enhance outcomes from cognitive training? A randomized controlled trial in healthy participants. Int. J. Neuropsychopharmacol. 16, 1927-1936. doi: 10.1017/S1461145713000539

McNab, F., and Klingberg, T. (2008). Prefrontal cortex and basal ganglia control access to working memory. Nat. Neurosci. 11, 103-107. doi: 10.1038/nn2024

Melby-Lervåg, M., Redick, T. S., and Hulme, C. (2016). Working memory training does not improve performance on measures of intelligence or other measures of "Far Transfer": evidence from a meta-analytic review. Perspect. Psychol. Sci. 11, 512-534. doi: 10.1177/1745691616635612

Mezger, E., Rauchmann, B. S., Brunoni, A. R., Bulubas, L., Thielscher, A., Werle, J., et al. (2019). Effects of prefrontal cathodal tDCS on brain glutamate levels and resting state connectivity: a randomized, sham-controlled, cross-over trial in healthy volunteers. Clin. Neurophysiol. 131:e127. doi: 10.1016/j.clinph.2019.12.310

Möller, A., Nemmi, F., Karlsson, K., and Klingberg, T. (2017). Transcranial electric stimulation can impair gains during working memory training and affects the resting state connectivity. Front. Hum. Neurosci. 11:364. doi: 10.3389/fnhum.2017.00364

Morrison, A. B., and Chein, J. M. (2011). Does working memory training work? The promise and challenges of enhancing cognition by training working memory. Psychon. Bull. Rev. 18, 46-60. doi: 10.3758/s13423-010-0034-0 
Nissim, N. R., O’Shea, A., Indahlastari, A., Telles, R., Richards, L., Porges, E., et al. (2019). Effects of in-scanner bilateral frontal tDCS on functional connectivity of the working memory network in older adults. Front. Aging Neurosci. 11:51. doi: 10.3389/fnagi.2019.00051

Owen, A. M., McMillan, K. M., Laird, A. R., and Bullmore, E. (2005). N-back working memory paradigm: a meta-analysis of normative functional neuroimaging studies. Hum. Brain Mapp. 25, 46-59. doi: 10.1002/hbm.20131

Palva, J. M., Monto, S., Kulashekhar, S., and Palva, S. (2010). Neuronal synchrony reveals working memory networks and predicts individual memory capacity. Proc. Natl. Acad. Sci. U. S. A. 107, 7580-7585. doi: 10.1073/pnas.0913113107

Peña-Gómez, C., Sala-Lonch, R., Junqué, C., Clemente, I. C., Vidal, D., Bargalló, N., et al. (2012). Modulation of large-scale brain networks by transcranial direct current stimulation evidenced by resting-state functional MRI. Brain Stimul. 5, 252-263. doi: 10.1016/j.brs.2011.08.006

Polania, R., Nitsche, M. A., and Paulus, W. (2011). Modulating functional connectivity patterns and topological functional organization of the human brain with transcranial direct current stimulation. Hum. Brain Mapp. 32, 1236-1249. doi: 10.1002/hbm.21104

Power, J. D., Mitra, A., Laumann, T. O., Snyder, A. Z., Schlaggar, B. L., and Petersen, S. E. (2014). Methods to detect, characterize, and remove motion artifact in resting state fMRI. Neurolmage 84, 320-341. doi: 10.1016/j. neuroimage.2013.08.048

Power, J. D., Schlaggar, B. L., and Petersen, S. E. (2015). Recent progress and outstanding issues in motion correction in resting state fMRI. NeuroImage 105, 536-551. doi: 10.1016/j.neuroimage.2014.10.044

Redick, T. S. (2019). The hype cycle of working memory training. Curr. Dir. Psychol. Sci. 28, 423-429. doi: 10.1177/0963721419848668

Reuter-Lorenz, P. A., and Park, D. C. (2010). Human neuroscience and the aging mind: a new look at old problems. J. Gerontol. B Psychol. Sci. Soc. Sci. 65, 405-415. doi: 10.1093/geronb/gbq035

Richmond, L., Wolk, D., Chein, J., and Olson, I. R. (2014). Transcranial direct current stimulation enhances verbal working memory training performance over time and near transfer outcomes. J. Cogn. Neurosci. 26, 2443-2454. doi: 10.1162/jocn_a_00657

Rushworth, M. F. S., Hadland, K. A., Gaffan, D., and Passingham, R. E. (2003). The effect of cingulate cortex lesions on task switching and working memory. J. Cogn. Neurosci. 15, 338-353. doi: 10.1162/089892903321593072

Saad, Z. S., Reynolds, R. C., Argall, B., Japee, S., and Cox, R. W. (2004). "SUMA: an interface for surface-based intra- and inter-subject analysis with AFNI" in 2004 2nd IEEE International Symposium on Biomedical Imaging: Nano to Macro; April 18, 2004; Arlington, USA, 1510-1513.

Sala, G., and Gobet, F. (2019). Cognitive training does not enhance general cognition. Trends Cogn. Sci. 23, 9-20. doi: 10.1016/j.tics.2018.10.004

Sala-Llonch, R., Peña-Gómez, C., Arenaza-Urquijo, E. M., Vidal-Piñeiro, D., Bargallo, N., Junque, C., et al. (2012). Brain connectivity during resting state and subsequent working memory task predicts behavioural performance. Cortex 48, 1187-1196. doi: 10.1016/j.cortex.2011.07.006

Sambataro, F., Murty, V. P., Callicott, J. H., Tan, H. Y., Das, S., Weinberger, D. R., et al. (2010). Age-related alterations in default mode network: impact on working memory performance. Neurobiol. Aging 31, 839-852. doi: 10.1016/j. neurobiolaging.2008.05.022

Sandrini, M., Fertonani, A., Cohen, L. G., and Miniussi, C. (2012). Double dissociation of working memory load effects induced by bilateral parietal modulation. Neuropsychologia 50, 396-402. doi: 10.1016/j.neuropsychologia. 2011.12.011
Saunders, N., Downham, R., Turman, B., Kropotov, J., Clark, R., Yumash, R., et al. (2015). Working memory training with tDCS improves behavioral and neurophysiological symptoms in pilot group with post-traumatic stress disorder (PTSD) and with poor working memory. Neurocase 21, 271-278. doi: 10.1080/13554794.2014.890727

Seeley, W. W., Menon, V., Schatzberg, A. F., Keller, J., Glover, G. H., Kenna, H., et al. (2007). Dissociable intrinsic connectivity networks for salience processing and executive control. J. Neurosci. 27, 2349-2356. doi: 10.1523/ JNEUROSCI.5587-06.2007

Shipstead, Z., Redick, T. S., and Engle, R. W. (2012). Is working memory training effective? Psychol. Bull. 138, 628-654. doi: 10.1037/a0027473

Simons, D. J., Boot, W. R., Charness, N., Gathercole, S. E., Chabris, C. F., Hambrick, D. Z., et al. (2016). Do "Brain-Training” programs work? Psychol. Sci. Public Interest 17, 103-186. doi: 10.1177/1529100616661983

Stephens, J. A., and Berryhill, M. E. (2016). Older adults improve on everyday tasks after working memory training and neurostimulation. Brain Stimul. 9, 553-559. doi: 10.1016/j.brs.2016.04.001

Stephens, J. A., Jones, K. T., and Berryhill, M. E. (2017). Task demands, tDCS intensity, and the COMT val158 met polymorphism impact tDCS-linked working memory training gains. Sci. Rep. 7:13463. doi: 10.1038/s41598-017-14030-7

Talsma, L. J., Kroese, H. A., and Slagter, H. A. (2017). Boosting cognition: effects of multiple-session transcranial direct current stimulation on working memory. J. Cogn. Neurosci. 29, 755-768. doi: 10.1162/jocn_a_01077

Teixeira-Santos, A. C., Moreira, C. S., Magalhaes, R., Magalhaes, C., Pereira, D. R., Leite, J., et al. (2019). Reviewing working memory training gains in healthy older adults: a meta-analytic review of transfer for cognitive outcomes. Neurosci. Biobehav. Rev. 103, 163-177. doi: 10.1016/j.neubiorev.2019.05.009

Trumbo, M. C., Matzen, L. E., Coffman, B. A., Hunter, M. A., Jones, A. P., Robinson, C. S., et al. (2016). Enhanced working memory performance via transcranial direct current stimulation: the possibility of near and far transfer. Neuropsychologia 93, 85-96. doi: 10.1016/j.neuropsychologia.2016.10.011

Unsworth, N., Heitz, R. P., Schrock, J. C., and Engle, R. W. (2005). An automated version of the operation span task. Behav. Res. Methods 37, 498-505. doi: 10.3758/bf03192720

Vatansever, D., Manktelow, A. E., Sahakian, B. J., Menon, D. K., and Stamatakis, E. A. (2017). Angular default mode network connectivity across working memory load. Hum. Brain Mapp. 38, 41-52. doi: 10.1002/hbm.23341

Wiegand, A., Nieratschker, V., and Plewnia, C. (2016). Genetic modulation of transcranial direct current stimulation effects on cognition. Front. Hum. Neurosci. 10:651. doi: 10.3389/fnhum.2016.00651

Wu, Y. J., Tseng, P., Huang, H. W., Hu, J. F., Juan, C. H., Hsu, K. S., et al. (2016). The facilitative effect of transcranial direct current stimulation on visuospatial working memory in patients with diabetic polyneuropathy: a pre-post sham-controlled study. Front. Hum. Neurosci. 10:479. doi: 10.3389/ fnhum.2016.00479

Conflict of Interest: The authors declare that the research was conducted in the absence of any commercial or financial relationships that could be construed as a potential conflict of interest.

Copyright (c) 2020 Cerreta, Mruczek and Berryhill. This is an open-access article distributed under the terms of the Creative Commons Attribution License (CC BY). The use, distribution or reproduction in other forums is permitted, provided the original author(s) and the copyright owner(s) are credited and that the original publication in this journal is cited, in accordance with accepted academic practice. No use, distribution or reproduction is permitted which does not comply with these terms. 